\title{
Elastic liposomes-in-vehicle formulations destined for skin therapy: The synergy between type of liposomes and vehicle
}

Zora Palac ${ }^{1}$, Julia Hurler ${ }^{2}$, Nataša Škalko-Basnet ${ }^{2}$, Jelena Filipović-Grčić ${ }^{1}$, Željka Vanić $^{1 *}$

${ }^{1}$ Department of Pharmaceutics, Faculty of Pharmacy and Biochemistry, University of Zagreb, 10000 Zagreb, Croatia

${ }^{2}$ Drug Transport and Delivery Research Group, Department of Pharmacy, Faculty of Health Sciences, University of Tromsø, 9037 Tromsø, Norway

Keywords: deformable liposomes, propylene glycol liposomes, hydrogel, cream, sustained release, texture analysis, hydrophilic drug, skin

*Correspondence: Željka Vanić

A. Kovačića 1, 10000 Zagreb, Croatia

Tel +3851 6394762

Fax +38514612691

Email: zeljka.vanic@pharma.hr 


\section{ABSTRACT}

Objective: The present study is focused on optimization of elastic liposomes-in-vehicle formulations in respect to drug release and formulation properties. By combining penetration potential of elastic liposomes containing high ratio of entrapped drug and physicochemical properties of vehicles, both affecting the release and texture properties, optimal formulation could be achieved.

Materials and methods: Deformable, propylene glycol-containing or conventional liposomes with hydrophilic model drug (diclofenac sodium) were incorporated into the following vehicles appropriate for skin application: a hydrogel, a cream base and derma membrane structure base cream (DMS base). Each formulation was assessed for in vitro drug release and mechanical properties.

Results and discussion: The composition and type of both liposomes and the vehicle affected the rate and amount of the released drug. The cream base exhibited the slowest release, followed by the hydrogel and DMS base. Similar release profiles were achieved with both types of elastic vesicles (deformable and propylene glycol liposomes); the slowest release was observed for conventional liposomes, regardless of the vehicle used. The drug release profiles from different liposomes-in-vehicle formulations were in agreement with the physicochemical properties of the formulations. All of the liposomes were found to be compatible with the hydrogel preserving its original textures, whereas a significant decrease in all texture parameters was observed for liposomes-in-DMS base, regardless of liposome type.

Conclusion: Propylene glycol liposomes-in-hydrogel is considered as the optimal formulation for improving skin delivery of hydrophilic drug. Further investigations involving in vivo animal studies are necessary to confirm its applicability in skin therapy. 


\section{INTRODUCTION}

Effective (trans)dermal drug delivery depends on both the selection of an appropriate drug and the properties of the delivery system (vehicle). Vehicles exert pronounced effects on the epidermis, affecting hydration, lubrication, drying, skin smoothness, occlusion and protection. In addition, the physicochemical properties of vehicles influence the pharmacokinetic properties of the incorporated drug by controlling its release from the vehicle and affecting penetration through the stratum corneum, permeation through the layers of skin and absorption into the blood. Therefore, interactions between the vehicle, skin and drug can influence the final therapeutic effect of the applied formulation ${ }^{1,2}$. For this reason, the selection of an appropriate vehicle is an important factor in increasing the efficacy of a topically applied drug ${ }^{3,4}$.

Despite the numerous types of available vehicles, dermal and particularly transdermal delivery of hydrophilic drugs is limited by the barrier nature of the outermost layers of the epidermis. Various strategies have been explored to enhance transdermal drug delivery, including adjustment of stratum corneum permeability by chemical penetration enhancers and electrical/external force methods, such as iontophoresis, sonophoresis and electroporation ${ }^{5}$. During the last two decades, considerable attention has been focused on developing nanodelivery systems that facilitate changes of the pharmacokinetic properties of a given drug and increase its bioavailability ${ }^{6}$. Because they are physiologically acceptable, phospholipid vesicles (liposomes) have shown great potential for improving the topical delivery of hydrophilic drugs. While conventional liposomes generally enhance skin deposition of the drug without affecting percutaneous permeation and transdermal delivery ${ }^{7}$, new classes of phospholipid vesicles, such as deformable (flexible, elastic) liposomes ${ }^{8-10}$, ethosomes ${ }^{11}$, invasomes $^{12}$ and propylene glycol-containing liposomes ${ }^{13}$, have been shown to increase the transport of active substances through the stratum corneum into the deeper layers of skin and 
even transdermally. Despite these advantages, topical application of liposomes can be restricted by their liquid nature. Thus, they must be incorporated into a suitable vehicle ${ }^{14}$. In addition to increasing the viscosity and preserving physical stability of conventional liposomes incorporated into hydrogels ${ }^{15-18}$, dermatological vehicles could affect drug bioavailability and play an important role in the care and maintenance of skin health ${ }^{2}$.

In the previous study ${ }^{19}$, we optimized the preparation conditions and evaluated the skin penetration potential of the two types of elastic liposomes containing a hydrophilic model drug (diclofenac sodium) using the stratum corneum-like pohospholipid vesicle-based permeation assay. Continuing this line of research, here we report on the optimization of semi-solid vehicles for liposomes. To the best of our knowledge, this is the first study which examines the influence of different types of vehicles on drug release and texture profiles of elastic liposomes in vehicle formulations. Deformable and propylene glycol as elastic liposomes and conventional liposomes (as control) were mixed into the following three vehicles: a hydrogel, a cream base (oil in water emulsion, $\mathrm{O} / \mathrm{W}$ ) and a novel cream-like vehicle that had a composition resembling natural human skin components. Each of these formulations (liposomes-in-vehicle) was tested for the drug release and characterized according to texture properties to optimize a formulation for topical administration of hydrophilic drug. 


\section{MATERIALS AND METHODS}

\subsection{Materials}

Soy lecithin (S 75) and derma membrane structure base cream classic (DMS base) were generous gifts from Lipoid GmbH (Ludwigshafen, Germany) and KOKO GmbH (Leichlingen, Germany), respectively. Carbopol $980 \mathrm{NF}$ and diclofenac sodium were kindly provided by BF Goodrich (Brussels, Belgium) and PLIVA Croatia Ltd. (Zagreb, Croatia), respectively. Sodium deoxycholate, Tween 60, glycerol monostearate, Myglyol $812 \mathrm{~N}$ and agarose were purchased from Sigma-Aldrich Company (St. Louis, USA). Propylene glycol, triethanolamine, cetylalcohol and vaselinum album were purchased from Kemig (Zagreb, Croatia). Ethanol and methanol were of analytical grade and obtained from Kemika (Zagreb, Croatia).

\subsection{Preparation and characterization of liposomes}

Deformable, propylene glycol and conventional liposomes were prepared by the film hydration method, followed by extrusion, as previously described ${ }^{19}$. Briefly, lipid components were dissolved in concentrated ethanol in a round bottomed flask. The ethanol was removed using a rotary vacuum evaporator (Büchi Rotavapor R-200, Büchi Labortechnik AG, Flawil, Switzerland) and the thin lipid film was hydrated with the aqueous drug solution. The concentrations of the lipid $(26 \mathrm{mM})$ and diclofenac sodium $(47 \mathrm{mM})$ were kept constant for all liposome preparations. Conventional liposomes were composed of S 75. The deformable liposomes consisted of S 75 and sodium deoxycholate (85/15, mass ratio), and the propylene glycol liposomes contained the same bilayer composition as conventional liposomes with an additional $10 \%(\mathrm{w} / \mathrm{w})$ propylene glycol in an aqueous phase. All of the liposomes were extruded three times through the $400-\mathrm{nm}$ pore size polycarbonate membranes (LiposoFast, Avestin, Canada). 
The mean diameters, size distributions (polydispersity) and zeta potentials of the liposomes were determined by photon correlation spectroscopy (Zetasizer 3000HS, Malvern Instruments, Malvern, UK) $)^{20,21}$. Membrane elasticity of extruded liposomes was evaluated by home-made device at an external pressure of 5 bar using 100-nm membranes. Detailed procedure with equation for calculation of the deformability degree (E) has been previously described in details ${ }^{14,19}$. Separation of the unentrapped drug was performed using gel chromatography on Sepharose CL-4B ${ }^{22}$. Thereafter, the liposomal fraction was concentrated using ultracentrifugation as previously described ${ }^{19}$. The concentrations of both the unentrapped (free) and liposome-entrapped drug were determined spectrophotometrically (Ultrospect Plus, Pharmacia LKB, Cambridge, UK). The concentrations of free diclofenac sodium were measured at $276 \mathrm{~nm}$ (demineralized water), and the entrapped drug was detected at $282 \mathrm{~nm}$ after the liposomes were dissolved in methanol.

\subsection{Preparation of semi-solid vehicles}

\subsubsection{Hydrogel}

Carbopol $980 \mathrm{NF}$ (0.5 g) was dispersed in demineralized water (88.5 g) using a magnetic stirrer (700 rpm) for 60 minutes. Propylene glycol (10 g) was then added, and the mixture neutralized by the addition of $50 \%(\mathrm{w} / \mathrm{w})$ triethanolamine in an amount sufficient to achieve a gel of $\mathrm{pH} 6^{23}$.

\subsubsection{Cream base}

The cream base was an oil-in-water $(\mathrm{O} / \mathrm{W})$ emulsion prepared using the modified procedure of Betz and coworkers ${ }^{24}$. Briefly, the lipophilic phase components [glycerol monostearate (4 g), cetylalcohol (6 g), Miglyol 812 (7.5 g), vaselinum album (25.5 g)] and hydrophilic phase components [Tween $60(6.5 \mathrm{~g})$, propylene glycol $(10 \mathrm{~g})$, distilled water $(40.5 \mathrm{~g})$ ] were separately heated to $60{ }^{\circ} \mathrm{C}$ using a water bath. The oil phase was then gradually poured into 
the water phase with constant stirring by hand to form an emulsion that was stirred until the cream reached room temperature. The formed $\mathrm{O} / \mathrm{W}$ emulsion was subjected to homogenization by a Three Roller Mill (Erweka SM/SMS, Erweka GmbH, Heusenstamm, Germany).

\subsection{Incorporation of liposomes into vehicles}

Each of the liposome suspensions (deformable, propylene glycol or conventional liposomes) containing only liposomally-entrapped diclofenac sodium and free of unentrapped drug, was gently mixed into the vehicles: hydrogel, cream base or DMS base.

The final concentration of liposomes in the vehicle was $10 \%$ (w/w, liposome suspension/vehicle). Control samples (solution of the free drug incorporated in vehicle, $10 \%$ w/w, drug solution/vehicle) contained the same concentration of the drug as in the liposomes.

\subsection{In vitro drug release studies}

The release of the drug from the liposomes incorporated into different vehicles was determined by the method originally introduced by Pesckha et al. ${ }^{25}$ and adapted for liposomal hydrogels ${ }^{14,16,23}$. Briefly, samples of the liposomes-in-vehicle formulations ( $3 \mathrm{~g}$ ) were placed in glass vials and separated from $5 \mathrm{~mL}$ of receptor solution (distilled water) by a $2 \%(\mathrm{w} / \mathrm{w})$ agarose layer, which permitted diffusion of both the released drug and intact liposomes from liposomes-in-vehicles (donor compartment). The vials were incubated at $32{ }^{\circ} \mathrm{C}$, and the receptor solution was completely replaced at specific time intervals. The amount of the drug released was determined spectrophotometrically (before and after the addition of methanol). Controls were examined under the same conditions.

The concentration of the drug was determined spectrophotometrically as described above. 


\subsection{Texture analysis of the liposomes-in-vehicle formulations}

A Texture Analyzer TA.XT Plus (Stable Micro Systems Ltd., Surrey, UK) was used to examine the texture properties of the liposomes-in-vehicle formulations. Fifty grams of each formulation was placed in a standard beaker, ensuring that no air bubbles were included and that the surface was smooth. A disk (40 mm in diameter) was pushed into the formulation (10 $\mathrm{mm}$ at a speed of $1 \mathrm{~mm} / \mathrm{s}$, respectively) and removed. Each formulation was measured five times, ensuring the same conditions for each measurement. The hardness of the each formulation was determined, and the texture properties, such as cohesiveness and adhesiveness, were calculated ${ }^{26}$. Control samples were examined under the same conditions.

\subsection{Statistical analysis}

Statistical analysis was performed using a one-way ANOVA, followed by Tukey's multiparametric post hoc test, and $\mathrm{p}<0.05$ was considered the minimum level of significance. Calculations were performed with the GraphPad Prism program, version 5.01 (GraphPad Software, Inc., San Diego, CA).

\section{RESULTS AND DISCUSSION}

\subsection{Characterization of liposomes}

Composition of the liposomes used as topical drug carrier is known to influence their physicochemical properties and therefore their efficacy as drug delivery system ${ }^{27,28}$. To allow deeper penetration of the hydrophilic drug into/through the skin, we prepared two types of elastic liposomes and compared them with each other and with conventional liposomes with respect to the following physicochemical properties: size, charge, membrane elasticity and entrapment capacity for diclofenac sodium (Table 1). The conventional and propylene glycol liposomes were nearly the same size and characterized by a homogenous size distribution, 
with a mean diameter between 155 (conventional liposomes) and $160 \mathrm{~nm}$ (propylene glycol liposomes), while the deformable liposomes were slightly smaller $(143 \mathrm{~nm})$ than the others. The presence of the edge activator (sodium deoxycholate) in the bilayers of deformable liposomes increased membrane flexibility and reduced surface tension, which are recognizable features of elastic liposomes, as shown in recent studies ${ }^{1421,29,30}$. Drug entrapment was quite low in the deformable liposomes $(60 \mu \mathrm{g}$ of the $\mathrm{drug} / \mathrm{mg}$ of lipid recovered), as compared to the propylene glycol liposomes (>140 $\mu \mathrm{g} / \mathrm{mg}$ of lipid). In addition, propylene glycol increased the degree of membrane elasticity, which was nearly equal to that of the deformable liposomes (Table 1). This result may be explained by the ability of propylene glycol to solubilize phospholipids and intercalate into the bilayer ${ }^{13,31,32}$, affecting the membrane flexibility of the vesicles. Regarding the surface charge, all of the liposomes had highly negative zeta potentials $(>-50 \mathrm{mV}$ ), indicating the formation of physically stable liposome suspensions, which was confirmed in the storage stability studies ${ }^{19}$.

\subsection{Liposomes-in-vehicle formulations}

Appropriate viscosity of liposomes used for skin application can be achieved by incorporation into dermatological vehicles ${ }^{15}$. Vehicle composition has been shown to influence the release properties of the incorporated drug and the penetration ability across/into the skin and may also improve the skin condition ${ }^{3,4,33}$. To examine the effect of the vehicle on the release of liposomally entrapped diclofenac sodium, different types of liposomes were incorporated into the following vehicles: the hydrogel, the cream base (O/W emulsion) and DMS base.

To follow the release of diclofenac sodium from the liposomes-in-vehicle formulations, we applied a so-called agarose method that has been shown to be very useful in evaluating drug release from liposomal vaginal gels ${ }^{14,16,23}$. The porosity of the agarose matrix permitted both intact liposomes and released (free) drug to diffuse through the matrix into the receptor 
solution, and the amount of drug released from the vehicle was determined spectrophotometrically before and after the addition of methanol to the supernatant over the agarose matrix ${ }^{16}$. With this method, the amount of intact liposomes released from the vehicle into the receptor medium can be determined, which is not feasible using the Franz diffusion cell method. Moreover, this method provides insight into the possible mechanisms of drug release from liposomes-in-vehicle formulations, which have been proposed to be based on the following four interconnected processes: leakage of the drug inside the vehicle, diffusion of the released drug to the receptor compartment, diffusion of intact liposomes from the vehicle into the receptor solution and release of the liposomally entrapped drug inside the receptor compartment $^{34}$.

The release of diclofenac sodium from the various liposomes-in-vehicle formulations is presented in Table 2 and Figure 1. The results (Table 2) confirm slower release of the drug from all of the liposomes-in-vehicle formulations compared with the control (solution of the drug in vehicle), and compared to the drug release from liposomal suspensions ${ }^{19}$. Differences in the drug release profiles were observed between all of the liposomes and vehicles examined. The slowest release of diclofenac sodium was observed for the cream base, followed by the hydrogel and DMS base (Table 2). Interestingly, release of the drug from liposomes-in-DMS base followed the same profile as corresponding control indicating possible release of the drug from all the liposomes upon their mixing into DMS base. A comparison of the drug-release profiles from different types of liposomes incorporated in hydrogel or cream base confirmed the slowest release from conventional liposomes in both cases; the drug release from propylene glycol liposomes and especially deformable vesicles was significantly faster $(\mathrm{p}<0.05)$. These results were in agreement with the previous study ${ }^{19}$, where the highest release of the drug was achieved from deformable, followed by propylene 
glycol-containing liposomes, while the slowest release was demonstrated by conventional liposomes.

The release of diclofenac sodium from all of the investigated formulations followed the Higuchi diffusion model ${ }^{35}$, which was confirmed by the good correlation coefficients ranging from 0.988 to 0.995 (Figure 1). This finding indicates that the rate-controlling step in the release process was the diffusion of the leaked drug through the vehicle network to the receptor medium, which was affected by both the composition of the phospholipid vesicles and the mechanical properties of the vehicles. Thus, after 24 hours of incubation of liposomes in hydrogel at $32{ }^{\circ} \mathrm{C}$, more than $35 \%$ of the drug was released from each formulation of elastic vesicles, while the lowest ratio of released drug (28\%) was observed for the conventional liposomes $(\mathrm{p}<0.05)$.

Although the fastest drug release profile from the liposomes-in-vehicle formulations was with DMS base, the highest release of the solution of the drug in vehicle (control) was achieved with the hydrogel $(p<0.05)$. These results were expected to some extent given the hydrophilic properties of both the drug and the hydrogel. Diclofenac sodium has a dissociation constant (pKa) of 3.9 at $25^{\circ} \mathrm{C}$ in water and a $\log \mathrm{P}$ of $1.45^{36}$. The hydrogel used in this study contained $89 \%$ water and $10 \%$ propylene glycol, which acts as both a humectant and a skin-penetration enhancer. Therefore, encapsulation into liposomes extended the release of the drug from the hydrogel.

An evaluation of intact liposome release from the hydrogel and the cream base showed a similar release ratio of both deformable and propylene glycol liposomes compared with conventional liposomes $(\mathrm{p}<0.05)$, which can be attributed to effect of the degree of membrane elasticity of the vesicles (Table 1) on the penetration of the liposomes through the vehicle and agarose matrix into the receptor solution (Table 2). Although propylene glycol liposomes had the same bilayer composition as conventional liposomes, they contained 
propylene glycol, a well-known skin-penetration enhancer ${ }^{37,38}$ that has been shown to contribute to membrane elasticity (Table 1), and subsequently affected the penetration of the liposomes through the vehicle network into the receptor medium. These results were consistent with those of a recent investigation ${ }^{14}$ that demonstrated the significance of vesicle composition on the drug-release profile from the hydrogel. Thus, liposomes with pronounced elastic properties diffused more rapidly through the hydrogel network than conventional liposomes ${ }^{14}$. Given that the intended route of administration of the drugs in liposomes-invehicle formulations is through the skin, penetration of the drug through the stratum corneum is of great importance. An assessment of the different classes of liposomes (deformable, propylene glycol and conventional liposomes) using a novel stratum corneum-like phospholipid vesicle-based permeation assay ${ }^{19}$ demonstrated that propylene glycol liposomes were the most promising formulation for improving the topical delivery of diclofenac sodium.

The success of dermatotherapy depends also on the properties of the formulation, particularly the spreading ability and retention of the formulation on the skin ${ }^{18}$. Texture analysis was used to evaluate the mechanical properties of the different liposomes-in-vehicle formulations, and parameters such as hardness, adhesiveness and cohesiveness were assessed (Figure 2). These properties have been directly correlated with administration parameters in vivo, such as spreadability (hardness) and retention of the formulation on the skin (adhesiveness) or its removal from the container (cohesiveness) ${ }^{14,26}$. Therefore, these investigations are of major importance during the development and optimization of formulations. In addition, they provide valuable data on the compatibility of the incorporated liposomes with the vehicle.

As shown in Figure 2, the texture characteristics were significantly different between all of the examined vehicles. The cream base showed highest values of all of the parameters assessed, followed by the hydrogel and DMS base. The cream base used in this study 
contained vaselinum album and cetylalcohol, which were responsible for the hardness of the formulation. Mixing the liposomes in the cream base significantly (ANOVA, p $<0.05$ ) lowered the initial hardness by approximately $30 \%$ for the conventional and propylene glycol liposomes and slightly more for the deformable liposomes (35\%) (Figure $2 \mathrm{~A}$ ).

Conventional liposomes have already been proven to be compatible with Carbopol gels ${ }^{15,23}$. Stability of liposomes incorporated in vehicle, especially emulsion and lamellar types of vehicle could be influenced by the vehicle's ingredients. DMS base is a novel cream-like vehicle used in corneotherapy that is prepared without classic emulsifiers and contains lipids similar to those found in human stratum corneum. It is composed of hydrogenated soy phosphatidylcholine, medium chain trigylcerides, phytosterols and squalane ${ }^{39}$. Clinical studies have confirmed increased levels of skin hydration and reparation of disrupted skin barriers with DMS base when used alone or with incorporated active ingredients ${ }^{39,40}$. Although it has been shown that liposomally encapsulated active compounds mixed in DMS base can significantly improve skin conditions (more than free active compounds in the vehicle) ${ }^{39}$, our results, which were based on texture analysis, indicate a possible physical instability of liposomes in DMS base. A nearly 2-fold decrease of the initial hardness was observed after incorporation of the liposomes into DMS base (more than 60\%, regardless of the type of liposome). In contrast, mixing a solution of the free drug (control) into DMS base reduced the initial hardness by only $20 \%$ (Figure 2A). Therefore, we speculate that there may be an interaction/incompatibility between all of the types of liposomes used in this study and DMS base, likely caused by the interaction of the negatively charged soy lecithin (S 75) liposomes with the ingredients of the DMS base, predominantly hydrogenated phospholipids. The $10 \%$ of liposomes in DMS base (w/w) represent relatively high source of the negative surface charge in the vehicle, probably causing the disturbance within the lamellar structure of DMS base. This assumption was also supported with release studies in which the ratio of intact 
liposomes released was lower than $1 \%$, even after 24 hours (Table 2). To verify the interactions/compatibility of liposomes with the components of the DMS vehicle, further studies based on hydrogenated soy phosphatidylcholine liposomes would be valuable.

The smallest decrease in the original hardness was observed with liposomes in the hydrogel. Thus, all liposomes are compatible with the hydrogel $(\mathrm{p}>0.05)$. These results are consistent with observations concerning Carbopol gels that were recently reported by Hurler and ŠkalkoBasnet $^{41}$ and Vanić and collaborators ${ }^{14}$, in which liposomes preserved the original structure of the gels. The other two texture parameters (cohesiveness and adhesiveness) followed similar patterns for the examined liposomes-in-vehicle formulations (Figure $2 \mathrm{~B}$ and $\mathrm{C}$ ).

Vehicle composition has been shown to play an important role in spreading and retaining the formulation at the application site $^{42}$ and in the release profile of the incorporated drug ${ }^{4}$. Phospholipid vesicles with pronounced elastic properties have been demonstrated to considerably improve transdermal delivery of hydrophilic drugs $s^{7,8,43}$. By combining penetration potential of elastic liposomes containing high ratio of entrapped drug and physicochemical properties of vehicles, both affecting the release and texture properties, optimal formulation could be achieved. Therefore, propylene glycol liposomes in a hydrogel is considered as the most promising formulation for improving topical delivery of the hydrophilic drug.

\section{CONCLUSION}

Deformable, propylene glycol and conventional liposomes containing diclofenac sodium were incorporated into different vehicles appropriate for skin application and characterized by in vitro drug release and texture parameters. The release of the drug from the different liposomes-in-vehicle formulations was affected by the composition of the vesicles and the mechanical properties of the vehicles. All types of liposomes preserved the original texture of 
hydrogel. Based on these studies, propylene glycol liposomes-in-hydrogel is the most suitable formulation due to the favorable physicochemical properties of liposomes, high trapping efficiency, the drug release profile from the vehicle, the ratio of intact liposomes released and the protective mechanism of the hydrogel on liposomes. However, to confirm the superiority of the proposed formulation for the skin therapy, animal studies would be beneficial.

\section{ACKNOWLEDGMENTS}

This work was supported by grant 006-0061117-1244 from the Ministry of Science, Education and Sports of the Republic of Croatia and project entitled "New approach in antimicrobial therapy: Biocompatible, biofilm-penetrating nanosystems" from the University of Zagreb. The authors thank to Lipoid (Ludwigshafen, Germany) for providing soy lecithin and KOKO GmbH (Leichlingen, Germany) for providing DMS base cream classic.

\section{DECLARATION OF INTEREST}

The authors report no declaration of interest.

\section{REFERENCES}

1. Daniels R, Knie U. Galenics of dermal product - vehicles, properties and drug release. J Dtsch Dermatol Ges 2007;5:369-383.

2. Weiss SC. Conventional topical delivery systems. Dermatol Ther 2011;24:471-476.

3. Rehman K, Zulfakar MH. Recent advances in gel technologies for topical and transdermal drug delivery. Drug Dev Ind Pharm 2014;40:433-440.

4. Sanna V, Peana AT, Moretti MD. Effect of vehicle on diclofenac sodium permeation from new topical formulations: in vitro and in vivo studies. Curr Drug Deliv 2009;6:93-100.

5. Delgado-Charro MB, Guy RH. Transdermal drug delivery. In: Hillery A.M., Lloyd A.W., Swarbrick J., eds. Drug Delivery and Targeting for Pharmacists and Pharmaceutical Scientists. London-New York: Taylor \& Francis, 2001:208-236. 
6. Bosselmann S, Williams III RO. Has nanotechnology led to improved therapeutic outcomes? Drug Dev Ind Pharm 2012;38:158-170.

7. Elsayed MMA, Abdallah OY, Naggar VF, Khalafallah NM. Lipid vesicles for skin delivery of drugs: Reviewing three decades of research. Int J Pharm 2007;332:1-16.

8. Cevc G, Gebauer D, Stieber J, Schatzlein A, Blume G. Ultraflexible vesicles, Transfersomes, have an extremely low pore penetration resistance and transport therapeutic amounts of insulin across the intact mammalian skin. Biochim Biophys Acta 1998;1368:201-215.

9. Cevc G, Blume G. New, highly efficient formulation of diclofenac for the topical, transdermal administration in ultradeformable drug carriers, Transfersomes. Biochim Biophys Acta 2001;1514:191-205.

10. Li S, Qiu Y, Zhang S, Gao Y. Enhanced transdermal delivery of 18 $\beta$-glycyrrhetic acid via elastic vesicles: in vitro and in vivo evaluation. Drug Dev Ind Pharm 2012;38:855865.

11. Touitou E, Dayan N, Bergelson L, Godin B, Eliaz M. Ethosomes - novel vesicular carriers for enhanced delivery: characterization and skin penetration properties. $\mathbf{J}$ Control Release 2000;65:403-418.

12. Dragicevic-Curic N, Scheglmann D, Albrecht V, Fahr A. Temoporfin-loaded invasomes: development, characterization and in vitro skin penetration studies. J Control Release 2008;127:59-69.

13. Manconi M, Mura S, Sinico C, Fadda AM, Vila AO, Molina F. Development and characterization of liposomes containing glycols as carriers for diclofenac. Colloid Surface A 2009;342:53-58.

14. Vanić Ž, Hurler J, Ferderber K, Golja Gašparović P, Škalko-Basnet N, Filipović-Grčić J. Novel vaginal drug delivery system: deformable propylene glycol liposomes-inhydrogel. J Liposome Res 2014;24:27-36.

15. Škalko N, Čajkovac M, Jalšenjak I. Liposomes with metronidazole for topical use: The choice of preparation method and vehicle. J Liposome Res 1998;8:283-293.

16. Pavelić Ž, Škalko-Basnet N, Jalšenjak I. Characterisation and in vitro evaluation of bioadhesive liposome gels for local therapy of vaginitis. Int J Pharm 2005;301:140148.

17. Mourtas S, Duraj S, Fotopoulou S, Antimisiaris SG. Integrity of liposomes in presence of various formulation excipients, when dispersed in aqueous media and in hydrogels. Colloids Surf B Biointerfaces 2008;61:270-276. 
18. Hurler J, Žakelj S, Mravljak J, Pajk S, Kristl A, Schubert R, Škalko-Basnet N. The effect of lipid composition and liposome size on the release properties of liposomesin-hydrogel. Int J Pharm 2013;456:49-57.

19. Palac Z, Engesland A, Flaten GE, Škalko-Basnet N, Filipović-Grčić J, Vanić Ž. Liposomes for (trans)dermal drug delivery: the skin-PVPA as a novel in vitro stratum corneum model in formulation development. J Liposome Res 2014; DOI: 10.3109/08982104.2014.899368.

20. Karn PR, Vanić Ž, Pepić I, Škalko-Basnet N. Mucoadhesive liposomal delivery systems: the choice of coating material. Drug Dev Ind Pharm 2011;37:482-488.

21. Vanić Ž, Hafner A, Bego M, Škalko-Basnet N 2013. The characterization of various deformable liposomes with metronidazole. Drug Dev Ind Pharm 39(03):481-488.

22. Vanić Ž, Barnert S, Süss R, Schubert R. Fusogenic activity of PEGylated pH-sensitive liposomes. J Liposome Res 2012;22:148-157.

23. Pavelić Ž, Škalko-Basnet N, Filipović-Grčić J, Martinac A, Jalšenjak I. Development and in vitro evaluation of a liposomal vaginal delivery system for acyclovir. J Control Release 2005;106:34-43.

24. Betz G, Aeppli A, Menshutina N, Leuenberger H. In vivo comparison of various liposome formulations for cosmetic application. Int J Pharm 2005;296:44-54.

25. Peschka R, Dennehy C, Szoka FC. A simple in vitro model to study the release kinetics of liposome encapsulated material. J Control Release 1998;56:41-51.

26. Hurler J, Engesland A, Kermany BP, Škalko-Basnet N. Improved texture analysis for hydrogel characterization: Gel cohesiveness, adhesiveness and hardness. J Appl Polym Sci 2012;125:180-188.

27. Šentjurc M, Vrhovnik K, Kristl J. Liposomes as a topical delivery system: the role of size on transport studied by the EPR imaging method. J Control Release, 1999;59:8797.

28. Caddeo C, Teskač K, Sinico C, Kristl J. Effect of resveratrol incorporated in liposomes on proliferation and UV-B protection of cells. Int J Pharm, 2008;363:183191.

29. Gillet A, Compere P, Lecomte F, Hubert P, Ducat E, Evrard B, Piel G. Liposome surface charge influence on skin penetration behaviour. Int J Pharm 2011;411:223231. 
30. Gillet A, Lecomte F, Hubert P, Ducat E, Evrard B, Piel G. Skin penetration behaviour of liposomes as a function of their composition. Eur J Pharm Biopharm 2011;79:4353.

31. Pavelić Ž, Škalko-Basnet N, Jalšenjak I. Liposomes containing drugs for treatment of vaginal infections. Eur J Pharm Sci 1999;8:345-351.

32. Pavelić Ž, Škalko-Basnet N, Schubert R. Liposomal gels for vaginal drug delivery. Int J Pharm 2001;219:139-149.

33. Nagelreiter C, Raffeiner S, Geyerhofer C, Klang V, Valenta C. Influence of drug content, type of semi-solid vehicle and rheological properties on the skin penetration of the model drug fludrocortisone acetate. Int J Pharm 2013;448:305-312.

34. Vanić Ž, Škalko-Basnet N. Nanopharmaceuticals for improved topical vaginal therapy: Can they deliver? Eur J Pharm Sci 2013;50:29-41.

35. Higuchi T. Rate of release of medicaments from ointment bases containing drugs in suspension. J Pharm Sci 1961;50:874-875.

36. Rainsford KD, Kean WF, Ehrlich GE. Review of the pharmaceutical properties and clinical effects of the topical NSAID formulation diclofenac epolamine. Curr Med Res Opin 2008;24:2967-2992.

37. Lane ME. Skin penetration enhancers. Int J Pharm 2013;447:12-21.

38. Schneider IM, Dobner B, Neubert R, Wohlrab W. Evaluation of drug penetration into human skin ex vivo using branched fatty acids and propylene glycol. Int J Pharm 1996;145:187-196.

39. Lautenschlager H. Biodegradable lamellar systems in skin care, skin protection and dermatology. SOFW-Journal 2013;139:2-8.

40. Szepietowski JC, Szepietowski T, Reich A. Efficacy and tolerance of the cream containing structured physiological lipids with endocannabinoids in the treatment of uremic pruritus: a preliminary study. Acta Dermatovenerol Croat 2005;13:97-103.

41. Hurler J, Škalko-Basnet N. Potentials of chitosan-based delivery systems in wound therapy: bioadhesion study. J Funct Biomater 2012;3:37-40.

42. Jones DS, Woolfson AD, Brown AF. Textural analysis and flow rheometry of novel, bioadhesive antimicrobial oral gels. Pharm Res 1997;14:450-457.

43. Cevc G, Blume G. Lipid vesicles penetrate into intact skin owing to the transdermal osmotic gradients and hydration force. Biochim Biophys Acta 1992;1104:226-232. 
Table 1. Physicochemical properties of liposomes

\begin{tabular}{lccc}
\hline Parameter & DL & PGL & CL \\
\hline Mean diameter (nm) & $143.3 \pm 5.1$ & $159.7 \pm 3.4$ & $156.1 \pm 2.6$ \\
Polydispersity index & $0.28 \pm 0.05$ & $0.20 \pm 0.07$ & $0.19 \pm 0.02$ \\
Zeta potential (mV) & $-56.3 \pm 0.7$ & $-61.4 \pm 1.1$ & $-58.4 \pm 0.8$ \\
$\begin{array}{l}\text { Degree of } \\
\text { deformability, E }\end{array}$ & $5.58 \pm 0.33$ & $5.23 \pm 0.31$ & $1.08 \pm 0.11$ \\
$\begin{array}{l}\text { Entrapment } \\
\text { (drug/lipid, } \mu \mathrm{g} / \mathrm{mg})\end{array}$ & $60.3 \pm 2.8$ & $142.8 \pm 4.2$ & $129.4 \pm 3.5$ \\
\hline
\end{tabular}

DL - deformable liposomes; $\quad$ PGL - propylene glycol liposomes;

$\mathrm{CL}$ - conventional liposomes

The values denote the mean \pm S.D. $(n=3)$. 
Table 2. In vitro release of diclofenac sodium from various liposomes-in-vehicle formulations

\begin{tabular}{|c|c|c|c|c|c|c|c|c|c|c|c|c|}
\hline \multirow{2}{*}{$\begin{array}{c}\mathrm{t} \\
\text { (h) }\end{array}$} & \multicolumn{4}{|c|}{ Hydrogel } & \multicolumn{4}{|c|}{ Cream base } & \multicolumn{4}{|c|}{ DMS base } \\
\hline & DL & PGL & $\mathrm{CL}$ & Control & DL & PGL & $\mathrm{CL}$ & Control & $\mathrm{DL}$ & PGL & $\mathrm{CL}$ & Control \\
\hline \multirow{3}{*}{1} & $7.1 \pm 0.7^{\mathrm{a}}$ & $4.2 \pm 0.4^{\mathrm{a}}$ & $1.6 \pm 0.7^{\mathrm{a}}$ & \multirow{3}{*}{$13.9 \pm 0.7$} & $2.8 \pm 0.3^{\mathrm{a}}$ & $2.5 \pm 0.2^{\mathrm{a}}$ & $1.9 \pm 0.2^{\mathrm{a}}$ & \multirow{3}{*}{$4.4 \pm 0.5$} & $7.8 \pm 0.9^{\mathrm{a}}$ & $7.5 \pm 0.7^{\mathrm{a}}$ & $7.2 \pm 0.0^{\mathrm{a}}$ & \multirow{3}{*}{$8.1 \pm 1.2$} \\
\hline & $7.5 \pm 0.6^{b}$ & $4.7 \pm 0.8^{b}$ & $2.0 \pm 0.7^{b}$ & & $3.1 \pm 0.3^{b}$ & $2.6 \pm 0.2^{b}$ & $2.0 \pm 0.2^{\mathrm{b}}$ & & $7.9 \pm 0.9^{b}$ & $7.8 \pm 0.6^{b}$ & $7.3 \pm 0.1^{b}$ & \\
\hline & $0.4 \pm 0.2^{c}$ & $0.5 \pm 0.2^{c}$ & $0.4 \pm 0.1^{\mathrm{c}}$ & & $0.3 \pm 0.1^{\mathrm{c}}$ & $0.1 \pm 0.1^{\mathrm{c}}$ & $0.1 \pm 0.1^{\mathrm{c}}$ & & $0.1 \pm 0.1^{\mathrm{c}}$ & $0.3 \pm 0.1^{\mathrm{c}}$ & $0.1 \pm 0.1^{\mathrm{c}}$ & \\
\hline \multirow{3}{*}{2} & $12.8 \pm 1.1^{\mathrm{a}}$ & $9.0 \pm 0.5^{\mathrm{a}}$ & $4.0 \pm 1.3^{\mathrm{a}}$ & \multirow{3}{*}{$22.7 \pm 1.2$} & $6.9 \pm 0.5^{\mathrm{a}}$ & $5.2 \pm 0.2^{\mathrm{a}}$ & $5.0 \pm 0.4^{\mathrm{a}}$ & \multirow{3}{*}{$\begin{array}{l}11.5 \pm \\
1.2\end{array}$} & $13.4 \pm 4.2^{\mathrm{a}}$ & $13.8 \pm 0.9^{\mathrm{a}}$ & $14.0 \pm 0.1^{\mathrm{a}}$ & \multirow{3}{*}{$14.6 \pm 2.0$} \\
\hline & $14.0 \pm 1.6^{\mathrm{b}}$ & $10.0 \pm 0.4^{\mathrm{b}}$ & $4.7 \pm 1.2^{\mathrm{b}}$ & & $7.9 \pm 1.1^{b}$ & $5.9 \pm 0.1^{\mathrm{b}}$ & $5.4 \pm 0.6^{b}$ & & $13.7 \pm 3.2^{b}$ & $14.0 \pm 3.1^{\mathrm{b}}$ & $14.3 \pm 0.4^{\mathrm{b}}$ & \\
\hline & $1.2 \pm 0.3^{\mathrm{c}}$ & $1.0 \pm 0.2^{\mathrm{c}}$ & $0.7 \pm 0.2^{\mathrm{c}}$ & & $1.0 \pm 0.6^{\mathrm{c}}$ & $0.7 \pm 0.1^{\mathrm{c}}$ & $0.4 \pm 0.1^{\mathrm{c}}$ & & $0.3 \pm 0.1^{c}$ & $0.2 \pm 0.1^{\mathrm{c}}$ & $0.3 \pm 0.1^{\mathrm{c}}$ & \\
\hline \multirow{3}{*}{4} & $16.9 \pm 2.2^{\mathrm{a}}$ & $14.2 \pm 0.3^{\mathrm{a}}$ & $7.1 \pm 2.2^{\mathrm{a}}$ & \multirow{3}{*}{$35.0 \pm 1.4$} & $11.1 \pm 0.3^{\mathrm{a}}$ & $9.0 \pm 0.4^{\mathrm{a}}$ & $7.4 \pm 0.3^{\mathrm{a}}$ & \multirow{3}{*}{$\begin{array}{l}22.0 \pm \\
3.1\end{array}$} & $24.6 \pm 0.7^{\mathrm{a}}$ & $25.0 \pm 0.6^{\mathrm{a}}$ & $27.8 \pm 0.2^{\mathrm{a}}$ & \multirow{3}{*}{$25.6 \pm 2.3$} \\
\hline & $20.3 \pm 1.9^{b}$ & $17.2 \pm 0.5^{b}$ & $8.8 \pm 2.0^{\mathrm{b}}$ & & $12.8 \pm 0.7^{b}$ & $10.0 \pm 0.1^{\mathrm{b}}$ & $8.0 \pm 0.4^{b}$ & & $24.9 \pm 3.4^{b}$ & $25.3 \pm 3.9^{b}$ & $28.2 \pm 0.7^{b}$ & \\
\hline & $3.4 \pm 0.6^{c}$ & $3.0 \pm 0.2^{c}$ & $1.7 \pm 0.2^{\mathrm{c}}$ & & $1.7 \pm 0.5^{\mathrm{c}}$ & $1.0 \pm 0.2^{\mathrm{c}}$ & $0.6 \pm 0.3^{c}$ & & $0.3 \pm 0.2^{c}$ & $0.3 \pm 3.1^{\mathrm{c}}$ & $0.4 \pm 0.1^{\mathrm{c}}$ & \\
\hline \multirow{3}{*}{6} & $20.7 \pm 0.9^{\mathrm{a}}$ & $17.5 \pm 0.1^{\mathrm{a}}$ & $8.7 \pm 1.9^{\mathrm{a}}$ & \multirow{3}{*}{$44.8 \pm 2.3$} & $14.5 \pm 1.4^{\mathrm{a}}$ & $12.5 \pm 0.4^{\mathrm{a}}$ & $10.3 \pm 0.2^{\mathrm{a}}$ & \multirow{3}{*}{$\begin{array}{l}31.1 \pm \\
4.2\end{array}$} & $37.4 \pm 4.1^{\mathrm{a}}$ & $38.5 \pm 3.7^{\mathrm{a}}$ & $38.7 \pm 2.9^{\mathrm{a}}$ & \multirow{3}{*}{$39.2 \pm 1.9$} \\
\hline & $28.0 \pm 1.9^{b}$ & $24.0 \pm 0.7^{b}$ & $11.8 \pm 2.1^{\mathrm{b}}$ & & $19.9 \pm 0.7^{b}$ & $17.3 \pm 0.3^{b}$ & $12.1 \pm 0.5^{b}$ & & $27.8 \pm 4.1^{b}$ & $39.1 \pm 3.1^{b}$ & $39.2 \pm 2.6^{\mathrm{b}}$ & \\
\hline & $7.3 \pm 0.7^{\mathrm{c}}$ & $6.5 \pm 0.5^{c}$ & $3.1 \pm 0.2^{\mathrm{c}}$ & & $5.4 \pm 0.7^{\mathrm{c}}$ & $4.8 \pm 0.2^{c}$ & $1.8 \pm 0.4^{\mathrm{c}}$ & & $0.4 \pm 0.1^{c}$ & $0.6 \pm 3.2^{c}$ & $0.5 \pm 0.2^{c}$ & \\
\hline \multirow{3}{*}{24} & $37.4 \pm 1.4^{\mathrm{a}}$ & $35.5 \pm 1.7^{\mathrm{a}}$ & $28.5 \pm 1.8^{\mathrm{a}}$ & \multirow{3}{*}{$89.3 \pm 2.2$} & $28.5 \pm 2.6^{\mathrm{a}}$ & $25.8 \pm 1.7^{\mathrm{a}}$ & $20.4 \pm 0.9^{\mathrm{a}}$ & \multirow{3}{*}{$\begin{array}{l}64.2 \pm \\
2.4\end{array}$} & $75.6 \pm 2.1^{\mathrm{a}}$ & $80.3 \pm 3.4^{\mathrm{a}}$ & $79.3 \pm 4.3^{\mathrm{a}}$ & \multirow{3}{*}{$76.9 \pm 7.1$} \\
\hline & $49.1 \pm 1.0^{\mathrm{b}}$ & $45.8 \pm 0.9^{b}$ & $32.3 \pm 1.7^{\mathrm{b}}$ & & $35.8 \pm 2.1^{\mathrm{b}}$ & $32.8 \pm 1.4^{\mathrm{b}}$ & $24.1 \pm 1.1^{b}$ & & $76.1 \pm 2.9^{b}$ & $80.9 \pm 3.8^{b}$ & $80.5 \pm 4.1^{b}$ & \\
\hline & $11.7 \pm 0.8^{\mathrm{c}}$ & $11.3 \pm 0.7^{\mathrm{c}}$ & $3.8 \pm 0.4^{\mathrm{c}}$ & & $7.3 \pm 0.5^{c}$ & $7.0 \pm 0.4^{\mathrm{c}}$ & $3.7 \pm 0.4^{\mathrm{c}}$ & & $0.5 \pm 0.2^{\mathrm{c}}$ & $0.6 \pm 0.3^{c}$ & $0.8 \pm 0.3^{\mathrm{c}}$ & \\
\hline
\end{tabular}

DL - deformable liposomes; $\quad$ PGL - propylene glycol liposomes; CL - conventional liposomes; ${ }^{\text {a }}$ free drug released $(\%) ;{ }^{b}$ total drug released $(\%) ;{ }^{\mathrm{c}}$ drug in intact liposomes released (\%)

Samples of liposomes-in-vehicles containing diclofenac sodium were incubated at $32{ }^{\circ} \mathrm{C}$ in distilled water (for details, see section 2.5 .). The amount of the drug in the release media was determined before (free drug) and after (total drug) disruption of the released liposomes with methanol. The difference between the total and the free drug released indicates the amount of the drug still present in the liposomes and released from the vehicle into the receptor solution (intact liposomes with drug). Control experiments (vehicles containing free drug, instead of liposomes) were performed simultaneously. The values denote the mean \pm S.D. $(n=3)$. 
A

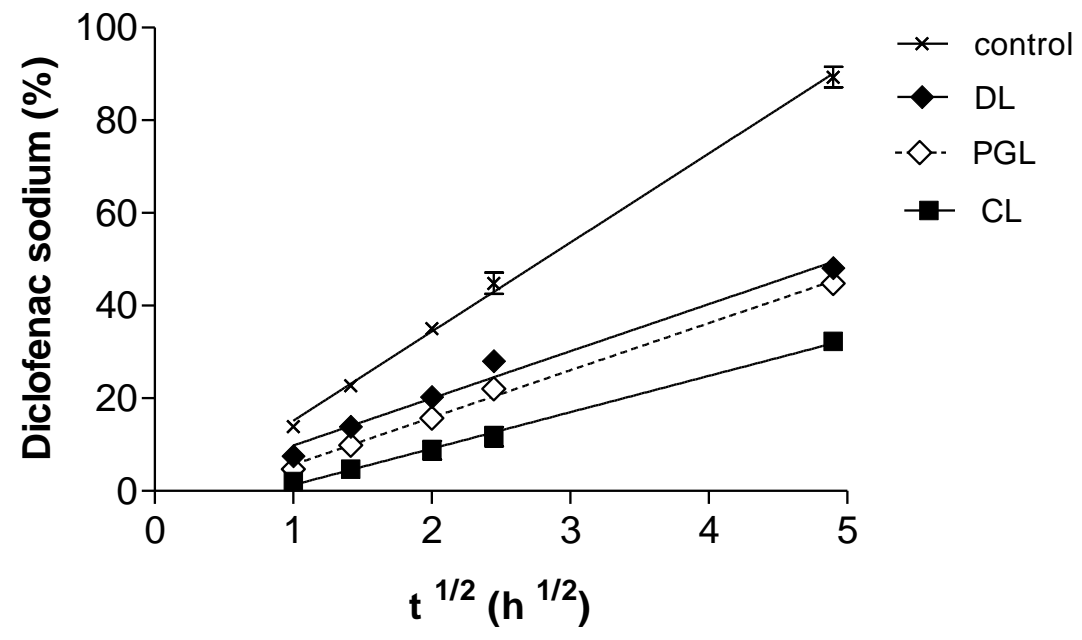

B

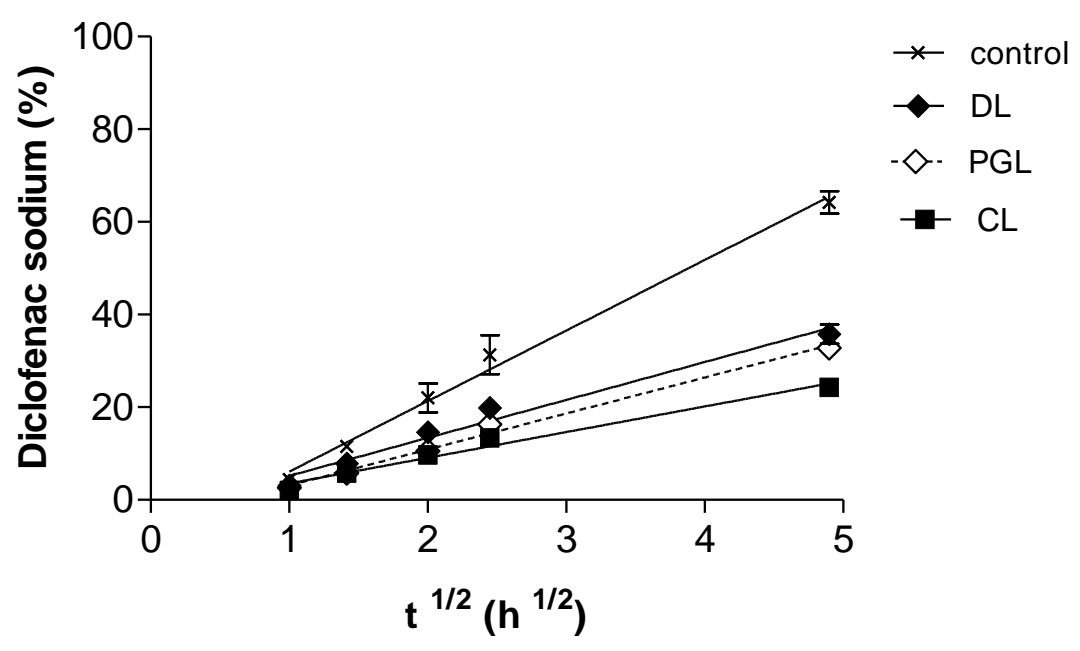

C

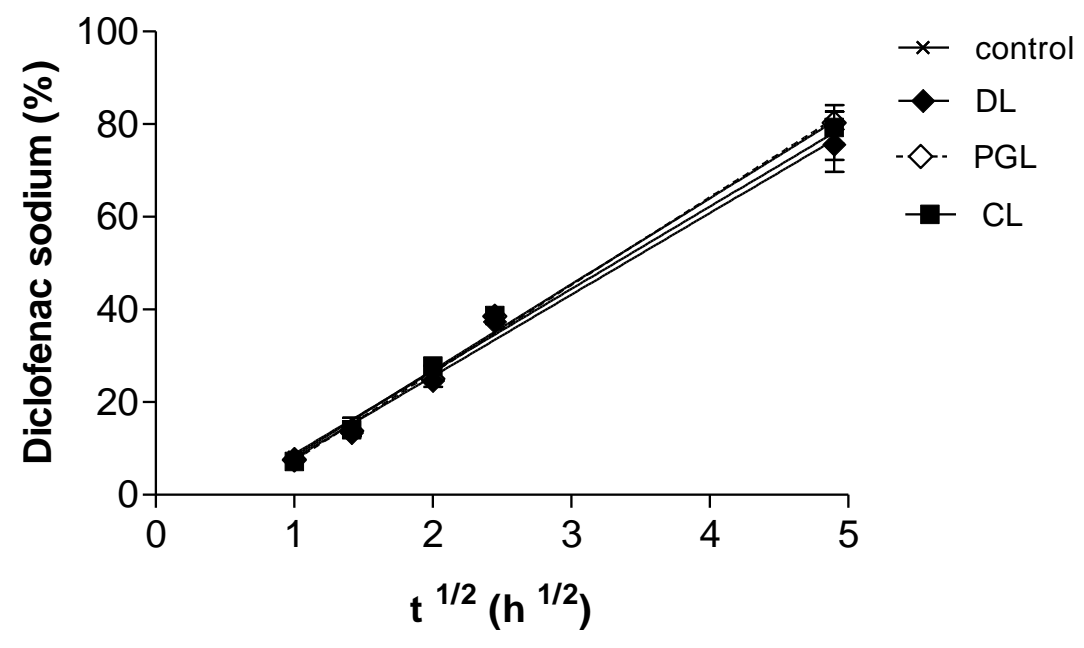

Figure 1. 

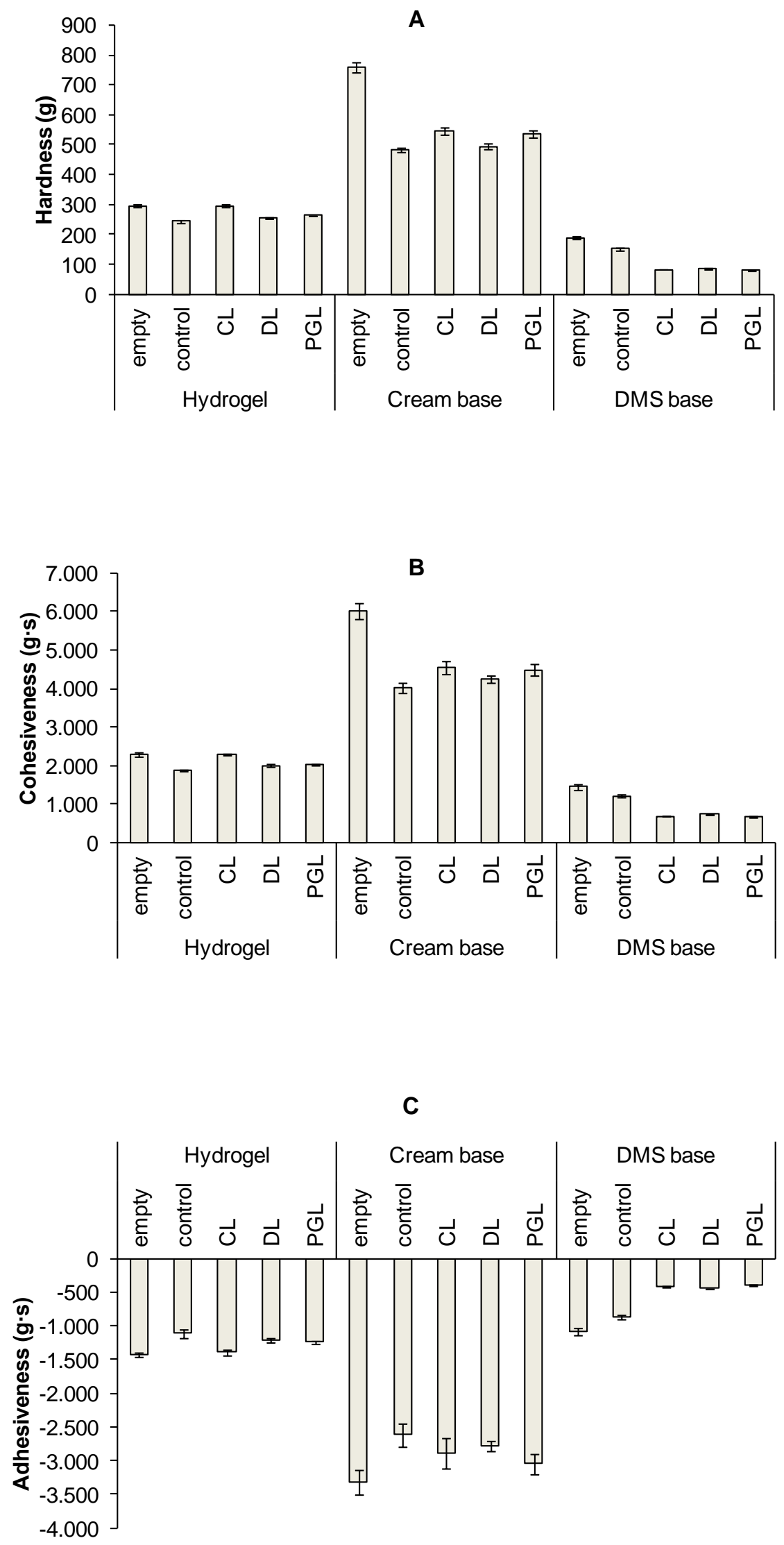

Figure 2. 


\section{FIGURE CAPTIONS:}

Figure 1. Cumulative release of diclofenac sodium from various liposomes-in-vehicle formulations plotted according to Higuchi ${ }^{35}$ equation: liposomes-in-hydrogel (A), liposomesin-cream base (B), liposomes-in-DMS base (C). The amounts of the released drug were detected in receptor media before addition of methanol (DL - deformable liposomes; PGL propylene glycol liposomes; $\mathrm{CL}$ - conventional liposomes). The values denote the mean \pm S.D. $(n=3)$. The values without error-bars have standard deviation equal or smaller than the size of the symbols.

Figure 2. Influence of liposome types on the texture properties of vehicles: hardness (A), cohesiveness (B) and adhesiveness (C). Empty sample denotes original vehicle, while control sample indicates a solution of the drug incorporated in vehicle. CL, DL and PGL represent different types of liposomes in vehicle. Concentration of the free drug and liposomes in vehicles was kept constant for all the samples $(10 \% \mathrm{w} / \mathrm{w}$, solution of free drug or liposomes/vehicle). The values indicate the mean \pm S.D $(n=5)$. 